\title{
A Note on Counting Homomorphisms of Paths
}

\author{
Roger B. Eggleton • Michał Morayne
}

Received: 25 October 2010 / Revised: 25 October 2012 / Published online: 24 November 2012 (C) The Author(s) 2012. This article is published with open access at Springerlink.com

\begin{abstract}
We obtain two identities and an explicit formula for the number of homomorphisms of a finite path into a finite path. For the number of endomorphisms of a finite path these give over-count and under-count identities yielding the closed-form formulae of Myers. We also derive finite Laurent series as generating functions which count homomorphisms of a finite path into any path, finite or infinite.
\end{abstract}

Keywords Path · Homomorphism - Generating function - Catalan number . Endomorphism

Mathematics Subject Classification (2010) 05A15 (primary). 60G50 (secondary)

\section{Introduction}

Path homomorphisms appear in many contexts in combinatorics, semigroup theory and the theory of stochastic processes. In this note we count homomorphisms of a finite path into any path. Our formulae for the number of endomorphisms of a finite path overcount or under-count by a sum involving weighted central binomial coefficients; using an identity recently proved by Lin and Zeng [3], we note that each yields the elegant closed-form formulae of Myers [4]. For homomorphisms of a finite path into a finite path we use a result of Sato and Cong [5] to obtain an explicit enumeration formula.

R. B. Eggleton

Department of Mathematics, Illinois State University, Normal, IL 61790, USA

e-mail: roger@ilstu.edu

M. Morayne ( $\square)$

Institute of Mathematics and Computer Science, Wrocław University of Technology,

Wybrzeże Wyspiańskiego 27, 50-370 Wrocław, Poland

e-mail: michal.morayne@pwr.wroc.pl 
We also obtain relevant generating function expressions that yield, as a particular case, the recent result obtained by Arworn and Wojtylak [2] for counting finite path homomorphisms.

\section{Notation and Terminology}

A graph $X$ has vertex set $V(X)$ and edge set $E(X)$. Given two graphs $G$ and $H$, a mapping $f: V(G) \rightarrow V(H)$ is a homomorphism from $G$ to $H$ if $\{f(u), f(v)\} \in$ $E(H)$ whenever $\{u, v\} \in E(G)$. We write $f: G \rightarrow H$ and denote the set of all homomorphisms from $G$ to $H$ by $\operatorname{Hom}(G, H)$.

Let the two-way infinite path $P_{\infty}$ be the graph with vertices all integers $i$ and edges all pairs $\{i, i+1\}$. The one-way infinite path $P_{\infty}^{+}$and the finite path $P_{n}$ of length $n$ are defined by restricting the vertices to nonnegative integers or to nonegative integers less then $n+1$, respectively. (We follow the convention that $P_{n}$ has $n$ edges and $n+1$ vertices.) In this paper $G$ will always be the finite path $P_{n}$, and $H$ will be a finite or infinite path.

Let $a, b \in V(H)$, possibly with $a=b$. Then we write

$$
\operatorname{Hom}\left(P_{n}, H ; a, b\right)=\left\{f \in \operatorname{Hom}\left(P_{n}, H\right): f(0)=a, f(n)=b\right\},
$$

and

$$
\operatorname{Hom}\left(P_{n}, H ; a\right)=\bigcup_{b \in V(H)} \operatorname{Hom}\left(P_{n}, H ; a, b\right)
$$

The cardinalities of these families are denoted by

$$
\pi\left(P_{n}, H ; a, b\right)=\left|H o m\left(P_{n}, H ; a, b\right)\right|, \quad \pi\left(P_{n}, H ; a\right)=\left|H o m\left(P_{n}, H ; a\right)\right| .
$$

Of course,

$$
\pi\left(P_{n}, H ; a\right)=\sum_{b \in V(H)} \pi\left(P_{n}, H ; a, b\right) .
$$

We also define the potentially infinite cardinality

$$
\pi\left(P_{n}, H\right)=\sum_{a \in V(H)} \pi\left(P_{n}, H ; a\right) .
$$

\section{Homomorphisms of $\boldsymbol{P}_{\boldsymbol{n}}$ into $\boldsymbol{P}_{\boldsymbol{r}}$}

For $r \geq 1$, consider homomorphisms $f: P_{n} \rightarrow P_{r}$. Evidently

$\pi\left(P_{n}, P_{r} ; a, 0\right)=\pi\left(P_{n-1}, P_{r} ; a, 1\right)$ and $\pi\left(P_{n}, P_{r} ; a, r\right)=\pi\left(P_{n-1}, P_{r} ; a, r-1\right)$ 
and if $0<b<r$ then

$$
\pi\left(P_{n}, P_{r} ; a, b\right)=\pi\left(P_{n-1}, P_{r} ; a, b-1\right)+\pi\left(P_{n-1}, P_{r} ; a, b+1\right) .
$$

From (1) and (2), summation of $\pi\left(P_{n}, P_{r} ; a, b\right)$ over $0 \leq b \leq r$ gives

$$
\pi\left(P_{n}, P_{r} ; a\right)=2 \pi\left(P_{n-1}, P_{r} ; a\right)-\pi\left(P_{n-1}, P_{r} ; a, 0\right)-\pi\left(P_{n-1}, P_{r} ; a, r\right)
$$

and then

$$
\pi\left(P_{n}, P_{r}\right)=2 \pi\left(P_{n-1}, P_{r}\right)-\sum_{a=0}^{r} \pi\left(P_{n-1}, P_{r} ; a, 0\right)-\sum_{a=0}^{r} \pi\left(P_{n-1}, P_{r} ; a, r\right) .
$$

By symmetry,

$$
\pi\left(P_{n-1}, P_{r} ; a, 0\right)=\pi\left(P_{n-1}, P_{r} ; r-a, r\right)=\pi\left(P_{n-1}, P_{r} ; 0, a\right) .
$$

Hence (4) yields

$$
\pi\left(P_{n}, P_{r}\right)=2 \pi\left(P_{n-1}, P_{r}\right)-2 \pi\left(P_{n-1}, P_{r} ; 0\right),
$$

and iteration of (5) gives us

$$
\pi\left(P_{n}, P_{r}\right)=2^{n} \pi\left(P_{0}, P_{r}\right)-\sum_{k=1}^{n} 2^{k} \pi\left(P_{n-k}, P_{r} ; 0\right), \quad \pi\left(P_{0}, P_{r}\right)=r+1 .
$$

Hence we have the over-count identity:

Theorem 3.1 The number of homomorphisms from $P_{n}$ to $P_{r}$ satisfies the identity

$$
\pi\left(P_{n}, P_{r}\right)=2^{n}(r+1)-\sum_{k=1}^{n} 2^{k} \pi\left(P_{n-k}, P_{r} ; 0\right) .
$$

Since $\pi\left(P_{0}, P_{r} ; 0\right)=1$, iterating (3) with $a=0$ yields

$$
\pi\left(P_{n}, P_{r} ; 0\right)=2^{n}-\sum_{i=1}^{n} 2^{i-1} \pi\left(P_{n-i}, P_{r} ; 0,0\right)-\sum_{i=1}^{n} 2^{i-1} \pi\left(P_{n-i}, P_{r} ; 0, r\right) .
$$

With (6), the over-count identity (Theorem 3.1) yields

$$
2^{n}(r+1)-\pi\left(P_{n}, P_{r}\right)
$$




$$
\begin{aligned}
& =\sum_{i=1}^{n} 2^{i}\left(2^{n-i}-\sum_{j=1}^{n-i} 2^{j-1} \pi\left(P_{n-i-j}, P_{r} ; 0,0\right)-\sum_{j=1}^{n-i} 2^{j-1} \pi\left(P_{n-i-j}, P_{r} ; 0, r\right)\right) \\
& =2^{n} n-\sum_{i=1}^{n} \sum_{j=1}^{n-i} 2^{i+j-1}\left(\pi\left(P_{n-i-j}, P_{r} ; 0,0\right)+\pi\left(P_{n-i-j}, P_{r} ; 0, r\right)\right) .
\end{aligned}
$$

Writing $i+j=k$, we deduce the under-count identity:

Theorem 3.2 The number of homomorphisms from $P_{n}$ to $P_{r}$ satisfies the identity $\pi\left(P_{n}, P_{r}\right)=2^{n}(r-n+1)+\sum_{k=2}^{n} 2^{k-1}(k-1)\left(\pi\left(P_{n-k}, P_{r} ; 0,0\right)+\pi\left(P_{n-k}, P_{r} ; 0, r\right)\right)$.

Let us consider the over-count and under-count identities in the special case $r=n$, the case of endomorphisms $f: P_{n} \rightarrow P_{n}$. Catalan numbers (sequence A000108 in OEIS [6]) are given by

$$
C_{n}=\frac{1}{n+1}\left(\begin{array}{c}
2 n \\
n
\end{array}\right)
$$

and play a key role in counting endomorphisms of $P_{n}$. For example

$$
\pi\left(P_{2 n}, P_{2 n} ; 0,0\right)=C_{n} .
$$

We shall need the following elementary identity for weighted sums of Catalan numbers.

Lemma 3.3 For any positive integer $n$, let $s=\left\lfloor\frac{n-1}{2}\right\rfloor$ and $t=\left\lfloor\frac{n}{2}\right\rfloor$. Then

$$
2^{n-1} \sum_{k=0}^{s} \frac{1}{4^{k}} C_{k}=2^{n}-\left(\begin{array}{l}
n \\
t
\end{array}\right)
$$

Proof The identity is easily verified for $n=1,2$. Suppose it holds for every positive integer up to some particular value of $n$. We now show that it also holds for $n+2$, whence the claim follows by induction. By hypothesis,

$$
\begin{aligned}
2^{n+1} \sum_{k=0}^{s+1} \frac{1}{4^{k}(k+1)}\left(\begin{array}{c}
2 k \\
k
\end{array}\right) & =4\left[2^{n}-\left(\begin{array}{l}
n \\
t
\end{array}\right)\right]+\frac{2^{n+1}}{4^{s+1}(s+2)}\left(\begin{array}{c}
2 s+2 \\
s+1
\end{array}\right) \\
& =2^{n+2}-D(n),
\end{aligned}
$$


where

$$
D(n)=4\left(\begin{array}{l}
n \\
t
\end{array}\right)-\frac{2^{n-2 s-1}}{s+2}\left(\begin{array}{c}
2 s+2 \\
s+1
\end{array}\right) .
$$

There are two cases. If $n=2 s+1$ then $t=s$ and we have

$$
D(n)=D(2 s+1)=4\left(\begin{array}{c}
2 s+1 \\
s
\end{array}\right)-\frac{1}{s+2}\left(\begin{array}{c}
2 s+2 \\
s+1
\end{array}\right)=\left(\begin{array}{c}
2 s+3 \\
s+1
\end{array}\right)=\left(\begin{array}{c}
n+2 \\
t+1
\end{array}\right) .
$$

If $n=2 s+2$ then $t=s+1$ and we have

$$
D(n)=D(2 s+2)=4\left(\begin{array}{c}
2 s+2 \\
s+1
\end{array}\right)-\frac{2}{s+2}\left(\begin{array}{c}
2 s+2 \\
s+1
\end{array}\right)=\left(\begin{array}{c}
2 s+4 \\
s+2
\end{array}\right)=\left(\begin{array}{c}
n+2 \\
t+1
\end{array}\right) .
$$

Together these cases verify the induction.

When $r=n$ the second sum in (6) vanishes, because $\pi\left(P_{m}, P_{n} ; 0, n\right)=0$ when $0 \leq m<n$. Because of (7), the first sum becomes a weighted sum of Catalan numbers, since $\pi\left(P_{m}, P_{n} ; 0,0\right)=\pi\left(P_{m}, P_{m} ; 0,0\right)$ if $0 \leq m \leq n$, and this is zero when $m$ is odd. With $s=\left\lfloor\frac{n-1}{2}\right\rfloor$ equation (6) yields

$$
\pi\left(P_{n}, P_{n} ; 0\right)=2^{n}-\sum_{i=0}^{n-1} 2^{n-i-1} \pi\left(P_{i}, P_{n} ; 0,0\right)=2^{n}-\sum_{k=0}^{s} 2^{n-2 k-1} \frac{1}{k+1}\left(\begin{array}{c}
2 k \\
k
\end{array}\right) .
$$

Simplifying with Lemma 3.3 this becomes

$$
\pi\left(P_{n}, P_{n} ; 0\right)=\left(\begin{array}{c}
n \\
\lfloor n / 2\rfloor
\end{array}\right) .
$$

This result was obtained by Arworn in [1]. With (9) and Theorem 3.1 we have

Theorem 3.4 The number of endomorphisms of $P_{n}$ is given by

$$
\pi\left(P_{n}, P_{n}\right)=2^{n}(n+1)-\sum_{k=0}^{n-1} 2^{n-k}\left(\begin{array}{c}
k \\
\lfloor k / 2\rfloor
\end{array}\right) .
$$

Again the case $r=n$ of Theorem 3.2 yields the companion result:

Theorem 3.5 The number of endomorphisms of $P_{n}$ is given by

$$
\pi\left(P_{n}, P_{n}\right)=2^{n}+\sum_{k=0}^{s} 2^{n-2 k-1} \frac{n-2 k-1}{k+1}\left(\begin{array}{c}
2 k \\
k
\end{array}\right),
$$

where $s=\left\lfloor\frac{n-1}{2}\right\rfloor$. 
The equivalence of the expressions for $\pi\left(P_{n}, P_{n}\right)$ in Theorems 3.4 and 3.5 implies the following identity for weighted sums of central binomial coefficients and Catalan numbers (with a "correction" applying on the right when $n$ is odd):

Corollary 3.6 For any integer $n \geq 2$, let $t=\left\lfloor\frac{n}{2}\right\rfloor$ and $\varepsilon=n-2 t$. Then

$$
2^{n} \sum_{k=0}^{t-1} \frac{1}{4^{k}}\left(\begin{array}{c}
2 k \\
k
\end{array}\right)+n 2^{n-1} \sum_{k=0}^{t-1} \frac{1}{4^{k}} C_{k}=n 2^{n}-(n+1) \varepsilon C_{t} .
$$

Applying Lemma 3.3 to Corollary 3.6, we have

$$
2^{2 t-1} \sum_{k=0}^{t-1} \frac{1}{4^{k}}\left(\begin{array}{c}
2 k \\
k
\end{array}\right)=t\left(\begin{array}{c}
2 t \\
t
\end{array}\right)
$$

Since $2\left(\begin{array}{c}2 k+1 \\ k\end{array}\right)=\left(\begin{array}{c}2 k+2 \\ k+1\end{array}\right)$ this immediately implies

$$
2^{2 t-1} \sum_{k=0}^{t-1} \frac{1}{4^{k}}\left(\begin{array}{c}
2 k+1 \\
k
\end{array}\right)=(t+1)\left(\begin{array}{c}
2 t+1 \\
t
\end{array}\right)-2^{2 t} .
$$

Using (10) and (11) to evaluate the sum in Theorem 3.4, we obtain

Theorem 3.7 For any integer $n \geq 0$, let $t=\left\lfloor\frac{n}{2}\right\rfloor$ and $\varepsilon=n-2 t$. Then the number of endomorphisms of $P_{n}$ is

$$
\pi\left(P_{n}, P_{n}\right)=2^{n}(n+2)-(2 n+1+\varepsilon)\left(\begin{array}{l}
n \\
t
\end{array}\right) .
$$

This result is equivalent to the elegant closed-form results of Myers [4], but here is expressed as a single formula by incorporating the "correction" mediated by $\varepsilon$ when $n$ is odd. In their Lemma 7 and Proposition 8, Lin and Zeng [3] recently used induction to prove identities equivalent to (10) and (11), and thence deduced expressions for the number of endomorphisms of $P_{n}$ equivalent to those of Myers.

More generally, an explicit formula for $\pi\left(P_{n}, P_{r}\right)$ can be obtained by combining Theorem 3.2 with Theorem 7 of Sato and Cong [5], though the latter goes beyond integer arithmetic. For even $n>0$ we have

$$
\pi\left(P_{n}, P_{r} ; 0,0\right)=\frac{4}{r+2} \sum_{k=1}^{s}(2 \cos k \theta)^{n} \sin ^{2}(r+1) k \theta
$$

where $s=\lfloor(r+1) / 2\rfloor$ and $\theta=\pi /(r+2)$. Also, for $n \equiv r(\bmod 2)$,

$$
\pi\left(P_{n}, P_{r} ; 0, r\right)=\frac{4}{r+2} \sum_{k=1}^{s}(2 \cos k \theta)^{n} \sin k \theta \sin (r+1) k \theta
$$


In all other cases $\pi\left(P_{n}, P_{r} ; 0,0\right)$ and $\pi\left(P_{n}, P_{r} ; 0, r\right)$ are zero. Hence

Theorem 3.8 The number of homomorphisms from $P_{n}$ to $P_{r}$ can be expressed in the form

$$
\pi\left(P_{n}, P_{r}\right)=2^{n-1}(2 r-n+1)+S_{1}(n, r)+S_{2}(n, r),
$$

where, for $s=\left\lfloor\frac{r+1}{2}\right\rfloor$ and $\theta=\frac{\pi}{r+2}$, the sums $S_{1}$ and $S_{2}$ are

$$
S_{1}(n, r)=\frac{2^{n+1}}{r+2} \sum_{0<2 j<n-1} \sum_{1 \leq k \leq s}(n-2 j-1)(\cos k \theta)^{2 j} \sin ^{2}(r+1) k \theta,
$$

$S_{2}(n, r)=\frac{2^{n+1}}{r+2} \sum_{0 \leq 2 j<n-r-1} \sum_{1 \leq k \leq s}(n-r-2 j-1)(\cos k \theta)^{r+2 j} \sin k \theta \sin (r+1) k \theta$,

for $r<n$, and $S_{2}(n, r)=0$ otherwise.

\section{Generating Functions with End Effects}

The main objective of this section is to obtain a generating function to enumerate the homomorphisms $f: P_{n} \rightarrow P_{r}$. If $P$ is any path, the possible presence of endpoints in $P$ complicates the problem of determining the cardinality of subsets of $\operatorname{Hom}\left(P_{n}, P\right)$. We shall use finite Laurent series to treat all cases. (Finite Laurent series have a much wider applicability as generating functions which enumerate graph homomorphisms, but here we confine ourselves to the case of paths.)

When there are no endpoints, then $P=P_{\infty}$ and $\pi\left(P_{n}, P_{\infty} ; a, b\right)$ is the coefficient of $x^{b}$ in the generating function

$$
\Pi\left[P_{n}, P_{\infty} ; a\right](x)=x^{a}\left(x^{-1}+x\right)^{n}=\frac{\left(1+x^{2}\right)^{n}}{x^{n-a}} .
$$

Hence

$$
\pi\left(P_{n}, P_{\infty} ; a\right)=\Pi\left[P_{n}, P_{\infty} ; a\right](1)=2^{n} .
$$

When there is one endpoint, then $P=P_{\infty}^{+}$. We discuss this case in detail before the case $P=P_{r}$, since the influence of one endpoint on the generating function is simpler than the interactive effects of two endpoints. Clarifying the details for $P_{\infty}^{+}$will obviate the necessity to explain most of the intricacies which arise for $P_{r}$.

Evidently

$$
\Pi\left[P_{0}, P_{\infty}^{+} ; a\right](x)=x^{a} .
$$

For $n \geq 0$ we have the coefficient identities

$$
\pi\left(P_{n+1}, P_{\infty}^{+} ; a, 0\right)=\pi\left(P_{n}, P_{\infty}^{+} ; a, 1\right)
$$


and

$$
\pi\left(P_{n+1}, P_{\infty}^{+} ; a, b\right)=\pi\left(P_{n}, P_{\infty}^{+} ; a, b-1\right)+\pi\left(P_{n}, P_{\infty}^{+} ; a, b+1\right)
$$

for $b>0$, so the generating function satisfies the recurrence relation

$$
\Pi\left[P_{n+1}, P_{\infty}^{+} ; a\right](x)=\left(x^{-1}+x\right) \Pi\left[P_{n}, P_{\infty}^{+} ; a\right](x)-x^{-1} \pi\left(P_{n}, P_{\infty}^{+} ; a, 0\right) .
$$

When $n \leq a$ the fact that $P_{\infty}^{+}$has an endpoint at 0 is inconsequential, so

$$
\Pi\left[P_{n}, P_{\infty}^{+} ; a\right](x)=\Pi\left[P_{n}, P_{\infty} ; a\right](x)=x^{a}\left(x^{-1}+x\right)^{n}
$$

for $0 \leq n \leq a$. But, in general, $\Pi\left[P_{n}, P_{\infty} ; a\right](x)$ exceeds $\Pi\left[P_{n}, P_{\infty}^{+} ; a\right](x)$ by a finite Laurent series which expresses the boundary effect of the endpoint of $P_{\infty}^{+}$: for brevity we shall refer to the latter series as the endpoint correction. The endpoint correction is conveniently expressed in terms of the following basic family of finite Laurent series, indexed by integers $r \geq 0$ :

$$
\Lambda_{0}(x)=1 \text { and } \Lambda_{r}(x)=x^{-r}+x^{r} \text { when } r>0 \text {. }
$$

These series have several useful properties, including

$$
\Lambda_{r}(x) \Lambda_{s}(x)=\Lambda_{r+s}(x)+\Lambda_{r-s}(x) \text { when } r>s>0,
$$

and

$$
\Lambda_{r}^{n}(x)=\sum_{2 k \leq n}\left(\begin{array}{l}
n \\
k
\end{array}\right) \Lambda_{r(n-2 k)}(x) \text { when } r>0
$$

where the sum formally ranges over all integers $k$ such that $2 k \leq n$, but effectively the constraint $k \geq 0$ also applies since the binomial coefficients are 0 when $k<0$.

Theorem 4.1 Let $m=n-(a+1)$ and $s=\left\lfloor\frac{m}{2}\right\rfloor$. Then, for all integers $n \geq 0$,

$$
\Pi\left[P_{n}, P_{\infty}^{+} ; a\right](x)=x^{a}\left(x^{-1}+x\right)^{n}-x^{-1} \sum_{k \leq s}\left(\begin{array}{l}
n \\
k
\end{array}\right) \Lambda_{m-2 k}(x) .
$$

Proof When $0 \leq n \leq a$ the endpoint correction is 0 , since the sum is empty when $s<0$, and this agrees with what we already know is the generating function in this range. In particular,

$$
\Pi\left[P_{a}, P_{\infty}^{+} ; a\right](x)=\Pi\left[P_{a}, P_{\infty} ; a\right](x)=x^{a}\left(x^{-1}+x\right)^{a}=\left(1+x^{2}\right)^{a}
$$

shows that $\pi\left(P_{a}, P_{\infty}^{+} ; a, 0\right)=1$, and then the recurrence relation for the generating function shows that the endpoint correction is $x^{-1}$ when $n=a+1$, in agreement with 
our claim. So now suppose the claim holds as far as some particular integer $n>a$. It follows that

$$
\pi\left(P_{n}, P_{\infty}^{+} ; a, 0\right)=\left(\begin{array}{l}
n \\
u
\end{array}\right)-\left(\begin{array}{c}
n \\
u-1
\end{array}\right)
$$

where $u=\frac{1}{2}(n-a)$ and $n \equiv a(\bmod 2)$; otherwise, $\pi\left(P_{n}, P_{\infty}^{+} ; a, 0\right)=0$.

There are two cases depending on the parity of $n$. When $n \equiv a(\bmod 2)$ the recurrence relation shows that $\Pi\left[P_{n+1}, P_{\infty}^{+} ; a\right](x)$ has the claimed form provided

$$
\sum_{k \leq s^{\prime}}\left(\begin{array}{c}
n+1 \\
k
\end{array}\right) \Lambda_{m^{\prime}-2 k}(x)=\left(x^{-1}+x\right) \sum_{k \leq s}\left(\begin{array}{l}
n \\
k
\end{array}\right) \Lambda_{m-2 k}(x)+\pi\left(P_{n}, P_{\infty}^{+} ; a, 0\right)
$$

holds, where $m^{\prime}=n-a=m+1=2 u$ and $s^{\prime}=\left\lfloor\frac{m^{\prime}}{2}\right\rfloor=s+1=u$. Thus, we require

$$
\sum_{k \leq u}\left(\begin{array}{c}
n+1 \\
k
\end{array}\right) \Lambda_{2(u-k)}(x)=\left(x^{-1}+x\right) \sum_{k \leq u-1}\left(\begin{array}{l}
n \\
k
\end{array}\right) \Lambda_{2(u-k)-1}(x)+\left[\left(\begin{array}{l}
n \\
u
\end{array}\right)-\left(\begin{array}{c}
n \\
u-1
\end{array}\right)\right] .
$$

The right side rearranges to

$$
\begin{gathered}
\left(\begin{array}{l}
n \\
0
\end{array}\right) \Lambda_{2 u}(x)+\sum_{1 \leq k \leq u-1}\left[\left(\begin{array}{c}
n \\
k-1
\end{array}\right)+\left(\begin{array}{l}
n \\
k
\end{array}\right)\right] \Lambda_{2(u-k)}(x) \\
+2\left(\begin{array}{c}
n \\
u-1
\end{array}\right) \Lambda_{0}(x)+\left[\left(\begin{array}{l}
n \\
u
\end{array}\right)-\left(\begin{array}{c}
n \\
u-1
\end{array}\right)\right]
\end{gathered}
$$

With Pascal's identity and $\Lambda_{0}(x)=1$, this reduces to the left side. The case when $n-a$ is odd is similar but slightly easier, so we omit the details. Thus, the claim follows by induction.

Corollary 4.2 If $n \equiv a+b(\bmod 2)$ and $k=\frac{1}{2}(n+b-a)$, then

$$
\begin{gathered}
\pi\left(P_{n}, P_{\infty}^{+} ; a, b\right)=\left(\begin{array}{l}
n \\
k
\end{array}\right)-\left(\begin{array}{c}
n \\
k-b-1
\end{array}\right) \text { when } 0 \leq b<n-a \\
\pi\left(P_{n}, P_{\infty}^{+} ; a, b\right)=\left(\begin{array}{l}
n \\
k
\end{array}\right) \text { when } b \geq n-a .
\end{gathered}
$$

If $n \not \equiv a+b(\bmod 2)$, then $\pi\left(P_{n}, P_{\infty}^{+} ; a, b\right)=0$. 
Theorem 4.1 can also be written in a form which emphasizes the relationship between $\Pi\left[P_{n}, P_{\infty}^{+} ; a\right](x)$ and $\Pi\left[P_{n}, P_{\infty} ; a\right](x)$, as follows:

Corollary 4.3 Let $m=n-(a+1)$. Then

$$
\begin{array}{r}
\Pi\left[P_{n}, P_{\infty} ; a\right](x)=x^{a} \sum_{2 k \leq n}\left(\begin{array}{l}
n \\
k
\end{array}\right) \Lambda_{n-2 k}(x), \\
\Pi\left[P_{n}, P_{\infty} ; a\right](x)-\Pi\left[P_{n}, P_{\infty}^{+} ; a\right](x)=x^{-1} \sum_{2 k \leq m}\left(\begin{array}{l}
n \\
k
\end{array}\right) \Lambda_{m-2 k}(x) .
\end{array}
$$

We have just shown that the generating function $\Pi\left[P_{n}, P_{\infty}^{+} ; a\right](x)$ is a polynomial in $x$ expressed as the difference of two finite Laurent series: the first is the generating function enumerating homomorphisms of $P_{n}$ into $P_{\infty}$, and the second, the endpoint correction, cancels out the coefficients of negative powers of $x$ and reduces the coefficients of small positive powers of $x$, namely those with exponent $b<n-a$.

Now consider generating functions for enumerating homomorphisms of $P_{n}$ into the finite path $P_{r}$. To avoid degeneracy we require $r \geq 1$. Here the relevant generating function is $\Pi\left[P_{n}, P_{r} ; a\right](x)$, and $\pi\left(P_{n}, P_{r} ; a, b\right)$ is the coefficient of $x^{b}$. Evidently

$$
\Pi\left[P_{0}, P_{r} ; a\right](x)=x^{a} .
$$

For $n \geq 0$ we have

$$
\pi\left(P_{n+1}, P_{r} ; a, 0\right)=\pi\left(P_{n}, P_{r} ; a, 1\right), \pi\left(P_{n+1}, P_{r} ; a, r\right)=\pi\left(P_{n}, P_{r} ; a, r-1\right),
$$

and for $0<b<r$

$$
\pi\left(P_{n+1}, P_{r} ; a, b\right)=\pi\left(P_{n}, P_{r} ; a, b-1\right)+\pi\left(P_{n}, P_{r} ; a, b+1\right),
$$

so the generating function satisfies the recurrence relation

$$
\begin{aligned}
\Pi\left[P_{n+1}, P_{r} ; a\right](x)=( & \left.x^{-1}+x\right) \Pi\left[P_{n}, P_{r} ; a\right](x) \\
& \quad-x^{-1} \pi\left(P_{n}, P_{r} ; a, 0\right)-x^{r+1} \pi\left(P_{n}, P_{r} ; a, r\right) .
\end{aligned}
$$

When $n \leq \min \{a, r-a\}$, the fact that $P_{r}$ has endpoints at 0 and $r$ does not come into play, so

$\Pi\left[P_{n}, P_{r} ; a\right](x)=\Pi\left[P_{n}, P_{\infty} ; a\right](x)=x^{a}\left(x^{-1}+x\right)^{n}$ for $0 \leq n \leq \min \{a, r-a\}$.

In general, $\Pi\left[P_{n}, P_{r} ; a\right](x)$ falls short of $\Pi\left[P_{n}, P_{\infty} ; a\right](x)$ by a finite Laurent series which expresses the effects of the two endpoints of $P_{r}$; we call it the double endpoint correction.

Theorem 4.4 For any integer $s \geq 0$ let

$$
L(s)=n-(a+1)-s(r+2) \text { and } R(s)=n-(r-a+1)-s(r+2) .
$$


Then for any integer $n \geq 0$ we have

$$
\begin{aligned}
& \Pi\left[P_{n}, P_{r} ; a\right](x)=x^{a}\left(x^{-1}+x\right)^{n} \\
& -x^{-1} \sum_{s \geq 0} \sum_{2 k \leq L(2 s)}\left(\begin{array}{l}
n \\
k
\end{array}\right) \Lambda_{L(2 s)-2 k}(x)-x^{r+1} \sum_{s \geq 0} \sum_{2 k \leq R(2 s)}\left(\begin{array}{l}
n \\
k
\end{array}\right) \Lambda_{R(2 s)-2 k}(x) \\
& +x^{-1} \sum_{s \geq 0} \sum_{2 k \leq R(2 s+1)}\left(\begin{array}{l}
n \\
k
\end{array}\right) \Lambda_{R(2 s+1)-2 k}(x)+x^{r+1} \sum_{s \geq 0} \sum_{2 k \leq L(2 s+1)}\left(\begin{array}{l}
n \\
k
\end{array}\right) \Lambda_{L(2 s+1)-2 k}(x) .
\end{aligned}
$$

Proof As in the proof of Theorem 4.1, a routine but more complicated induction on $n$ establishes the claim. The salient points for the argument are as follows. In the recurrence relation for the generating function, $\pi\left(P_{n}, P_{r} ; a, r\right)=0$ unless $n \geq r-a$ and $n \equiv a+r(\bmod 2)$. For the inductive step, note that $L(s)$ and $R(s)$ both increase by 1 when $n$ is replaced by $n+1$. Then the multiplicative identities noted for $\Lambda_{r}(x)$ in the case of the one-way infinite path, together with Pascal's identity suffice to complete the argument, though the manipulation of the double sums is intricate. We omit the details.

We can also deduce an explicit formula for the coefficient $\pi\left(P_{n}, P_{r} ; a, b\right)$. The same result obtained by different techniques appears as Theorem 3.1 of Arworn and Wojtylak [2].

Corollary 4.5 Let $n \equiv a+b(\bmod 2)$ and $k=\frac{1}{2}(n-a+b)$. Then

$$
\begin{aligned}
\pi\left(P_{n}, P_{r} ; a, b\right)= & \left(\begin{array}{l}
n \\
k
\end{array}\right)-\sum_{s \geq 0}\left(\begin{array}{c}
n \\
k-A(s)
\end{array}\right)-\sum_{s \geq 0}\left(\begin{array}{c}
n \\
k-B(s)
\end{array}\right)+\sum_{s \geq 0}\left(\begin{array}{c}
n \\
k-C(s)
\end{array}\right) \\
& +\sum_{s \geq 0}\left(\begin{array}{c}
n \\
k-D(s)
\end{array}\right)
\end{aligned}
$$

where

$$
\begin{aligned}
& A(s)=s(r+2)+b+1, \quad B(s)=(s+1)(r+2)-a-1, \\
& C(s)=(s+1)(r+2)-a+b, \quad D(s)=(s+1)(r+2) .
\end{aligned}
$$

If $n \not \equiv a+b(\bmod 2)$, then $\pi\left(P_{n}, P_{r} ; a, b\right)=0$.

Acknowledgments We are grateful to Dr. James D. Mitchell for drawing our attention to the elegant formulae of Myers, residing in an editorial note in OEIS. The research of the second author was partially supported by MNiSW Grant NN 206184233.

Open Access This article is distributed under the terms of the Creative Commons Attribution License which permits any use, distribution, and reproduction in any medium, provided the original author(s) and the source are credited. 


\section{References}

1. Arworn, Sr.: An algorithm for the numbers of endomorphisms on paths. Discrete Math. 309 (2009), 94-103

2. Arworn, Sr., Wojtylak, P.: An algorithm for the number of path homomorphisms. Discrete Math. 309 (2009), 5569-5573

3. Lin, Z.C., Zeng, J.: On the number of congruence classes of paths. arXiv reprint 1112.4026v1 [math.CO]. 17 Dec 2011

4. Myers, J.: OEIS sequence A102699, editorial note, 23 Dec 2008

5. Sato, M., Cong, T.T.: The number of minimal lattice paths restricted by two parallel lines. Discrete Math. 43, 249-26 (1983)

6. The on-line encyclopedia of integer sequences. The OEIS Foundation. http://oeis.org/ 